\title{
1D metallic states at 2D transition metal dichalcogenide semiconductor heterojunctions
}

\author{
Sridevi Krishnamurthi (iD) ${ }^{1}$ and Geert Brocks ${ }^{1 凶}$
}

Two-dimensional (2D) lateral heterojunctions between different transition metal dichalcogenides (TMDCs) have been realized in recent years. Homogeneous semiconducting TMDC layers are characterized by a topological invariant, their in-plane electric polarization. It suggests the possibility of one-dimensional (1D) metallic states at heterojunctions where the value of the invariant changes. We study such lateral 2D TMDC junctions by means of first-principles calculations and show that 1D metallic states emerge even in cases where the different materials are joined epitaxially. We find that the metallicity does not depend on structural details, but, as the invariant is protected by spatial symmetry only, it can be upset by breaking the symmetry. Indeed, 1D chargeand spin-density wave instabilities appear spontaneously, making 2D TMDC heterojunctions ideal systems for studying 1D systems.

npj 2D Materials and Applications (2021)5:43; https://doi.org/10.1038/s41699-021-00224-1

\section{INTRODUCTION}

Lateral structures that are grown from two-dimensional (2D) materials arouse growing scientific attention, because of their potential to open a route towards truly 2D electronics. In-plane $p-n$ junctions and barrierless Schottky contacts between 2D compounds provide the basic building blocks of $2 \mathrm{D}$ electronic devices $^{1-3}$. Lateral heterojunctions between a variety of $2 \mathrm{D}$ semiconductors are realized since chemical vapor deposition techniques have enabled the growth of sharp one-dimensional (1D) interfaces between different 2D materials. Transition metal dichalcogenides (TMDCs) are a particularly versatile class of compounds, where 2D heterostructures of TMDCs with a similar crystal structure are grown routinely now ${ }^{4-6}$. 2D junctions between TMDCs with different crystal structures can also be produced $^{7}$, and even structures with a large lattice constant mismatch, such as graphene or $\mathrm{h}-\mathrm{BN}$ and $\mathrm{MoS}_{2}$, have been grown as lateral junctions ${ }^{8}$.

2D TMDCs, $M X_{2}$, with $M$ a (transition) metal and $X=S, S e$, and $\mathrm{Te}$, constitute an extensive family of compounds, covering (semi) metals and semiconductors, depending on their elemental composition and their crystal phase. The compounds with $\mathrm{M}=$ Mo, $\mathrm{W}$ and $\mathrm{X}=\mathrm{S}$, Se have attracted the most attention, as they are direct bandgap semiconductors with potential applications in optoelectronics, photovoltaics, and photocatalysis ${ }^{9-11}$. Not surprisingly then, so far the focus has been mainly on junctions made from these materials, their band alignments, and interface transport properties ${ }^{1-8}$.

What has been exploited much less is the notion that, in their most common structure, these semiconductors are materials that can be characterized by an interesting topological invariant, that is, their in-plane electric polarization ${ }^{12-15}$. Insulating or semiconducting TMDCs with transition metals from different elemental groups can have a different value for this invariant. Following the general topological arguments, this suggests that if one creates an in-plane heterostructure between two such TMDCs, then at the junction the bandgap closes, and a one-dimensional (1D) metallic state is formed at the junction. This reasoning should hold for any edge or junction that involves a sudden change in invariant.
Indeed, TMDC grain boundaries and edges are known to have 1D metallic states that are exclusively localized at the boundary or edge, which display electronic properties that are especially prominent in 1D systems, such as charge-density waves (CDWs), spin-density waves (SDWs), or Lüttinger liquid behavior ${ }^{16-23}$. As grain boundaries and edges constitute 1D extended defects, one might argue that the appearance of 1D states is necessarily connected to the rather drastic character of these defects.

However, the presence or absence of 1D states is dictated by the change in topological invariant, the bulk polarization, of the corresponding TMDCs. In this paper, we will show that they also appear in perfectly lattice-matched heterojunctions, and these states arise out of a difference in the bulk polarization at the junction. We will discuss examples from two different cases, a polar/polar junction and a polar/nonpolar junction. The topological invariant is protected by spatial symmetry, which means it can be upset by breaking symmetry. The latter can, for instance, happen through CDWs and SDWs leading to a charge ordering and/or spin ordering with increased periodicity, which frequently is accompanied by the emergence of a (small) bandgap.

There have been computational studies on interfaces of semiconducting polar 2D materials such as AIN or $\mathrm{ZnO}, \mathrm{SiC}$, and (functionalized) graphene/BN ${ }^{12,24,25}$, where these junctions have been found to be metallic. The junction between blue and black phosphorene has been predicted to have a CDW arising at the 1D metallic interface ${ }^{26}$. However, such junctions have not been produced yet, and indeed clean junctions between these materials may be difficult to realize experimentally. Considering the recent developments cited above, in particular in controlled growth techniques, junctions between TMDCs are experimentally much more accessible.

\section{RESULTS}

\section{Origin of the interface states}

The macroscopic polarization is a topological invariant for $2 \mathrm{D}$ insulators with $D_{3 h}$ symmetry ${ }^{15}$. All semiconducting TMDCs that have the $\mathrm{H}$ structure belong to this class. Following the modern 


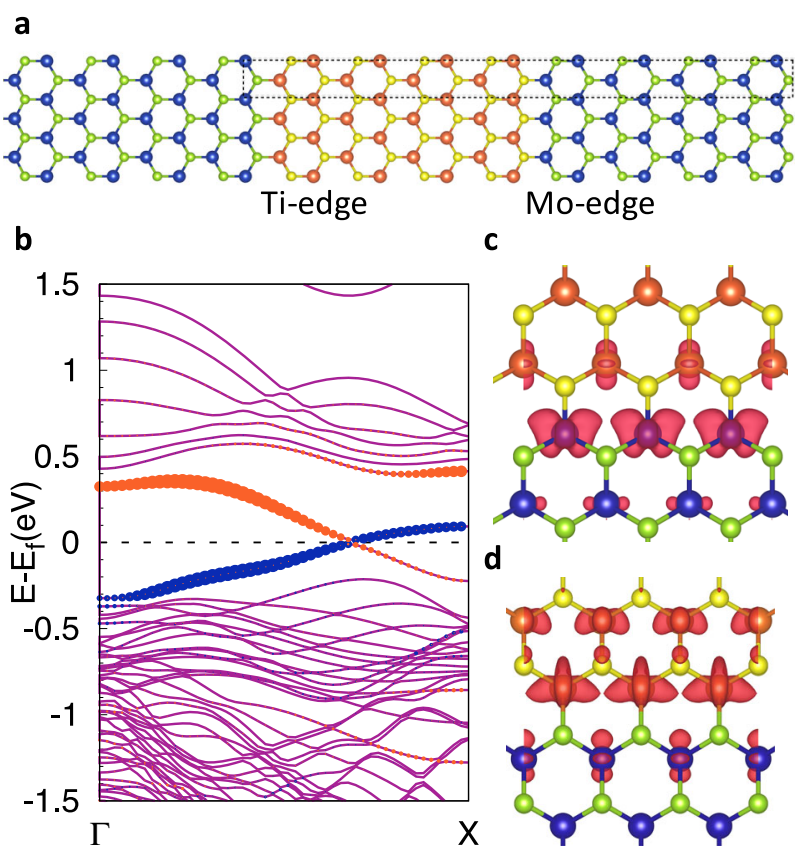

Fig. 1 The $1 \times$ polar-polar unit cell. a Heterojunctions of $\mathrm{H}-\mathrm{MoSe}_{2}$ and $\mathrm{H}-\mathrm{TiS}_{2}$; the blue, green, orange, and yellow spheres represent $\mathrm{Mo}, \mathrm{Se}, \mathrm{Ti}$, and $\mathrm{S}$ atoms, respectively; the black dotted lines indicate the supercell. The two structurally different junctions are labeled $\mathrm{Ti}$ edge and Mo edge. $\mathbf{b}$ The band structure of the supercell; the states highlighted in blue and red belong to Mo and Ti edges, respectively, identified by projecting the wave function densities on the Mo and $\mathrm{Ti}$ atoms at the edges. c Wave function density at the Mo edge at $E-E_{\mathrm{F}}=-0.2 \mathrm{eV}$. d Wave function density at the Ti edge at $E-E_{\mathrm{F}}=$ $+0.2 \mathrm{eV}$.

theory of polarization, it is straightforward to calculate the 2D polarization from first principles as an integral of the Berry phase over the Brillouin zone ${ }^{27}$. For the $\mathrm{H}$ structure, the direction of the polarization is normal to the zigzag direction of the hexagonal atomic pattern. Semiconducting TMDCs with the T structure have zero polarization, as this structure is centrosymmetric.

Connecting two insulators with a different polarization at a junction results in a jump in the polarization, if the projection of the latter along the normal to the junction is nonzero. According to the theory of topological invariants, this must be accompanied by a closure of the bandgap at the junction or, in other words, the junction becomes metallic ${ }^{24-26,28}$. We call this a nontrivial junction. If one connects two insulators with the same polarization, or if the projection of the polarization along the surface normal is the same, then there is no change in the topological invariant. This leads to a trivial junction, which, in general, is insulating. In order to construct a nontrivial junction, we need to choose two TDMCs with different polarizations and connect them along a zigzag edge; an example is shown in Fig. 1a. We also select TMDCs whose in-plane lattice constants are reasonably well matched, as too much strain at the junction would make the structure unrealistic from an experimental point of view. In this paper, we will show a $\mathrm{H} / \mathrm{H}$ junction, which is of polar/polar type, and a $\mathrm{H} / \mathrm{T}$ junction, which is of polar/nonpolar type, as examples of nontrivial heterojunctions in TMDCs.

Going through a database of $2 \mathrm{D} \mathrm{TMDCs}^{29}$, we identify a list of semiconducting $\mathrm{H}$ - and T-phase compounds. We then optimize their lattice constants and calculate their bulk polarization. In the $\mathrm{H}$ phase, the entire group $\mathrm{VI} M X_{2}(\mathrm{M}=\mathrm{Mo}, \mathrm{W} ; \mathrm{X}=\mathrm{S}, \mathrm{Se}$, Te) family of semiconducting TMDCs has the same polarization lattice, $\mathbf{P}=$ $\left(p_{1} \mathbf{a}_{1}+p_{2} \mathbf{a}_{2}\right) e / \Omega$, with $\left(p_{1}, p_{2}\right)=\left(\alpha+n_{1}, \beta+n_{2}\right) ; n_{1,2}=0, \pm 1, \pm 2, \ldots$, where $\mathbf{a}_{1,2}$ are the lattice vectors of the primitive $2 \mathrm{D}$ unit cell, where $(a, \beta)$ has the value $(2 / 3,1 / 3) ; e$ is the elementary charge, and $\Omega$ is the unit cell area. Choosing compounds for the junction from this group, for example, a heterojunction between $\mathrm{MoS}_{2}$ and $\mathrm{WS}_{2}$, means that the polarization is continuous across the interface, which results in a trivial, that is, semiconducting interface. Several experiments and ab initio calculations have indeed confirmed that this junction is semiconducting with a type-II (staggered) band alignment ${ }^{1,3}$.

\section{Polar/polar TMDC junctions}

The group $V$ TMDCs $M_{2}(M=V, N b, T a ; X=S, S e, T e)$ all are metallic, which is due to the transition metals having one less valence electron compared to group VI transition metals. Group IV TMDCs in the $\mathrm{H}$ structure, such as $\mathrm{TiX}_{2}$, are again semiconducting, with a calculated polarization $\mathbf{P}$ with $(a, \beta)=(1 / 3,2 / 3)$ that is different from that of group $\mathrm{VI} M X_{2}$. As this means that the topological invariant of group IV TMDCs is different from that of group VI TMDCs, this suggests the possibility of creating a nontrivial, that is, a metallic junction between, for instance, $\mathrm{TiX}_{2}$ and $\mathrm{MX}_{2}$. The $\mathrm{T}$ structure of $\mathrm{TiX}_{2}$ is actually lower in energy than the $\mathrm{H}$ structure, and is not suitable, as it is metallic. However, we suggest that it may be possible to create the metastable $\mathrm{TiX}_{2} \mathrm{H}$ structure by a suitable choice of growth conditions, as likewise, it is possible to create the metastable metallic T structure of $\mathrm{MoS}_{2}$ by suitable growth conditions ${ }^{30}$.

To be specific, an $\mathrm{H}-\mathrm{TiS}_{2}$ monolayer has a calculated optimized lattice constant of $3.34 \AA$, which is within $1 \%$ of the optimized lattice constant of $3.31 \AA$ of $\mathrm{MoSe}_{2}$. Both compounds are semiconductors, with $\mathrm{TiS}_{2}$ having a calculated (indirect) bandgap of $0.7 \mathrm{eV}$ and $\mathrm{MoSe}_{2}$ having a (direct) bandgap of $1.6 \mathrm{eV}$. These two semiconductors form a type-II band alignment according to our density functional theory (DFT) calculations, which, apart from the topological considerations discussed above, would result in a semiconducting junction.

Figure 1a shows our supercell model for the $\mathrm{TiS}_{2} / \mathrm{MoSe}_{2}$ junction along the zigzag direction. There are two different junctions in the supercell, due to the periodic boundary conditions imposed in the calculations. We call these the Ti edge and Mo edge, respectively, according to the transition metal atoms closest to the junction. Choosing a stoichiometric Se-S termination at the interfaces, as shown in the figure, we optimize the lattice constants again over the whole structure. After relaxation, the original bond lengths and angles are actually retained, and the structure has a uniform lattice constant close to $3.34 \AA$.

Figure $1 \mathrm{~b}$ shows the calculated non-spin-polarized bands of this structure, using a $1 \times$ periodicity in the direction of the Mo and $\mathrm{Ti}$ edges. There is a clear bandgap, with two bands crossing the gap. The wave functions associated with these two bands are localized at the two junctions in the supercell, where one band can be assigned to the $\mathrm{Ti}$ edge and the other to the Mo edge. The two edges clearly are metallic, with the $\mathrm{Ti}$ edge band being $1 / 3$ occupied and the Mo edge band being 2/3 occupied.

The Mo edge state has dominant Mo $d_{x y}$ and $d_{z^{2}}$ character, see Fig. 1c, whereas the Ti edge state has foremost Ti $d_{x y}$ and $d_{x^{2}-y^{2}} /$ $d_{z^{2}}$ character, with some participation of the other metal atom's $d$ states, see Fig. 1d. As the chalcogen atoms do not contribute appreciably to these edge states, this would imply that changing the chalcogen atoms at the edges would not affect the electronic structure. Indeed, on changing the chalcogen terminations at the junctions from a stoichiometric S-Se to a nonstoichiometric Se-Se or S-S, no change in the occupation or the dispersion of the edge states is observed.

Both the existence of states localized at the junctions, with energies in the bandgap, as well as the occupancy of those states can be deduced from the 2D polarization of the materials involved and its topological character. Going from material 1 to material 2 across a junction, the polarization jumps from $\mathbf{P}_{1}$ to $\mathbf{P}_{2}$, which 
would result in a polarization line charge density at the junction $\lambda=\left(\mathbf{P}_{2}-\mathbf{P}_{1}\right) \cdot \hat{\mathbf{n}}$, where $\hat{\mathbf{n}}$ is the in-plane unit vector normal to the junction. For a macroscopic junction, this polarization charge has to be neutralized by a compensating line charge density $\lambda_{e}=-\lambda$, such as to avoid an intrinsic electric field generating a polar catastrophe ${ }^{21,31,32}$.

In the present case, the compensating charge can only be of electronic origin and resides in states near the Fermi level, inside the bandgap. Following the reasoning of topological invariants, going across a junction from an insulator with a certain value for the topological invariant to an insulator with a different value for that invariant, then at the junction the gap has to close, that is, the junction is metallic, where the metallicity is carried by states localized at the junction.

Going from $\mathrm{MoSe}_{2}$ to $\mathrm{TiS}_{2}$, this gives $\lambda_{e}=(2 e) /(3 a)$ at the Mo edge, where $a$ is the lattice constant along the edge, which means that the corresponding edge state has to be $1 / 3$ occupied by holes (taking spin degeneracy into account). At the Ti edge, going from $\mathrm{TiS}_{2}$ to $\mathrm{MoSe}_{2}$, we have $\lambda_{e}=-(2 e) /(3 a)$, and an edge state that is $1 / 3$ occupied by electrons.

This interpretation is in line with the three-dimensional case of junctions between insulating oxide perovskites, such as $\mathrm{LaAlO}_{3} /$ $\mathrm{SrTiO}_{3}$, where a 2DEG emerges at the interface between the two insulators ${ }^{31}$. The interface metallicity has been attributed to the abrupt change of the valence charge of the cation at the interface, from $\mathrm{La}^{3+}$ to $\mathrm{Ti}^{4+}$, causing a charge transfer of $0.5 e$ in order to avoid a polar catastrophe. The 2D heterojunction between AIN and $\mathrm{SiC}$ has been analyzed in similar terms, where the difference in formal charges between the cations and anions in the 2D III-V and the IV-IV materials drives the formation of a 1DEG at the interface, in order to avoid a polar catastrophe ${ }^{24}$.

Our TMDC case is more subtle because there is no ionic charge discontinuity at the interface. The formal charges on the cations and ions on both sides of the junctions are the same in their respective compounds, $\mathrm{Ti}^{4+}\left(\mathrm{S}^{2-}\right)_{2}$ and $\mathrm{Mo}^{4+}\left(\mathrm{Se}^{2-}\right)_{2}$. Moreover, the compounds have the same structure, and we also see no structural distortions at the junction. The difference in polarization between the two compounds is then of purely electronic origin, as is the metallicity of the junction.

Formally, the analysis of the metallic junction presented above only holds in the macroscopic limit, that is, in the case where one has one junction between two semi-infinite 2D materials. Nevertheless, in our supercell model, which comprises strips of six TMDC units wide, we apparently already have reached this asymptotic limit. We found that if one uses strips of four or fewer units, one still observes edge states, see Supplementary Fig. 3, but the edge states at the Mo and Ti edges interact across the strip. This causes a gap to open up, similar to hybridization between orbitals resulting in a bonding/antibonding splitting, which is used to describe chemical bonding.

We see no evidence for a residual electric field existing over the width of the ribbon. The electrostatic potential is approximately constant over the $\mathrm{TiS}_{2}$ and the $\mathrm{MoSe}_{2}$ regions and has a step at the interface, see Supplementary Fig. 1 . The electrons residing in the edge states have then fully compensated the polarization charge. This is in agreement with an earlier work that shows that an electric field is present only if there are no edge states to compensate for the differences in polarization ${ }^{25}$. The band structure and the dispersion of the edge states suggest that the latter are not bulk states driven up or down by an electric field, but new states created in the gap ${ }^{33}$.

\section{Polar/nonpolar TMDC junctions}

The T-phase structure of TMDCs has zero polarization because it has inversion symmetry. Most of the T-phase TMDCs are metallic compounds, but $\mathrm{HfS}_{2}$ and $\mathrm{HfSe}_{2}$ are semiconductors. Constructing a junction between one of these and a semiconducting $\mathrm{H}$-phase a
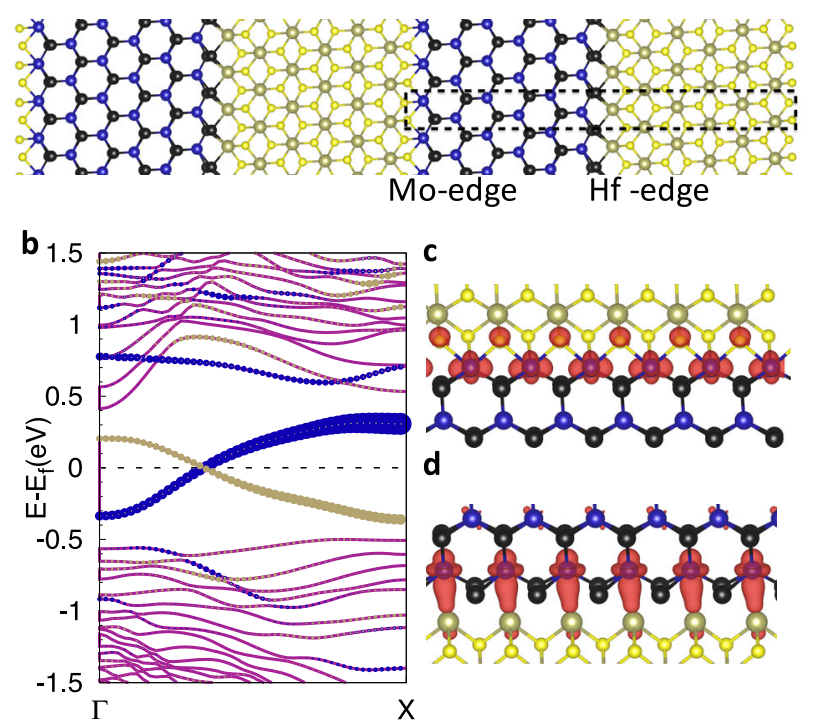

Fig. 2 The $1 \times$ polar-nonpolar unit cell. a Heterojunction of $\mathrm{H}$ $\mathrm{MoTe}_{2}$ and $\mathrm{T}-\mathrm{HfS}_{2}$; the blue, black, brown, and yellow spheres represent $\mathrm{Mo}, \mathrm{Te}, \mathrm{Hf}$, and $\mathrm{S}$ atoms, respectively; the black dotted lines indicate the supercell. $\mathbf{b}$ The band structure of the heterojunction; the states highlighted in blue and brown are states that belong to Mo and $\mathrm{Hf}$ edges, respectively, identified by projecting the wave function densities on the Mo and $\mathrm{Ti}$ atoms at the edges. c Wave function density at the Mo edge at $E-E_{\mathrm{F}}=-0.3 \mathrm{eV}$. d Wave function density at the $\mathrm{Hf}$ edge at $E-E_{\mathrm{F}}=-0.3 \mathrm{eV}$.

TMDC gives a topological discontinuity, which results in a metallic junction similar to that described in the previous section. The calculated lattice constant of $\mathrm{T}-\mathrm{HfS}_{2}$ is $3.62 \AA$, which is close to that of $\mathrm{H}-\mathrm{MoTe}_{2}, 3.56 \AA$, implying that a heterojunction between the two can be formed with minimal strain. The Perdew-Burke-Ernzerhof (PBE) bandgaps of $\mathrm{T}_{-} \mathrm{HfS} \mathrm{S}_{2}$ and $\mathrm{H}-\mathrm{MoTe}_{2}$ are 1.3 and $1.1 \mathrm{eV}$, respectively, and the band alignment between the two is type II, which, apart from polarization considerations, would imply a semiconducting junction. $\mathrm{T}-\mathrm{H}$ junctions cannot be stitched perfectly, with all metal atoms at the junctions having sixfold coordination by chalcogen atoms. In experimental work, one finds the $\beta$-junction structure ${ }^{7,34}$, where the metal atom on the T side of the junction (the $\mathrm{Hf}$ atom in this case) has sevenfold coordination, and the atom on the $\mathrm{H}$ side (the Mo atom) has sixfold coordination. We adopt this structure at one interface, calling it the Hf edge in the following. At the other interface, called the Mo edge, we construct a similar structure, giving the $\mathrm{Hf}$ atom a sixfold and the Mo atom a sevenfold coordination. Figure $2 \mathrm{a}$ shows the unit cell, where the two junctions are marked. More detailed images of the edge structures are given in Supplementary Fig. 4.

On optimizing, the lattice constant for the whole structure becomes $3.60 \AA$. The bond lengths involving atoms close to the interface undergo changes, while the atoms far away from the interface in both the $\mathrm{H}$ and $\mathrm{T}$ phase remain at their respective bulk positions.

The coordination at the edges does not influence the bulk polarization, of course. It also does not affect the fractional character of the polarization charges at the edges, as by adding or removing an atom one simply adds or removes an integer number of electrons. Therefore, edge metallicity is robust against atomic defects at the junction. To test this, we have also constructed a Mo edge structure where the $\mathrm{Hf}$ atom has a fivefold coordination and the Mo atom has a sixfold coordination, which gives a very similar electronic structure. Details can be found in Supplementary Fig. 5. 
The electronic structure of $\mathrm{H}-\mathrm{MoTe}_{2} / \mathrm{T}-\mathrm{HfS}_{2}$ heterojunctions is shown in Fig. 2b. It clearly displays a bandgap, with two bands, localized at the junctions, crossing in the gap at the Fermi level. The band from the $\mathrm{Hf}$ edge ( $\beta$-junction) is $2 / 3$ occupied, and its character is a combination of Mo and $\mathrm{Hf} d_{z^{2}}$ and $d_{x^{2}}$ orbitals, Fig. 2c. At the Mo edge, the band is $1 / 3$ occupied and has predominant Mo $d_{x y}$ and $d_{z^{2}}$ character, see Fig. $2 d$. The existence of these localized states and their occupancy follows from similar considerations as for the $\mathrm{H}-\mathrm{H}$ junctions discussed in the previous section.

\section{D electronic instabilities}

The metallic states at TMDC junctions clearly have a 1D character. Electron correlation and electron-lattice interactions are particularly effective in 1D systems and can perturb the metallic character. In the present case, the 1D states have an occupancy of $1 / 3$ or $2 / 3$, which suggests the possibility of a CDW and/or an SDW that triples the period in the direction along the junction. This would be similar to the edges of a TMDC flake or a TMDC grain boundary that display a $3 \times$ periodicity $^{16,17,22,23,35}$.

We start from the $\mathrm{H}-\mathrm{TiS}_{2} / \mathrm{H}-\mathrm{MoSe}_{2}$ junction, triple our supercell along the direction of the junction, and reoptimize the structure, using the PBE functional. The structure in the $3 \times$ cell does actually not change as compared to the $1 \times$ structure, meaning that we do not observe a Peierls distortion. Figure $3 a$ shows the $3 \times$ band

a

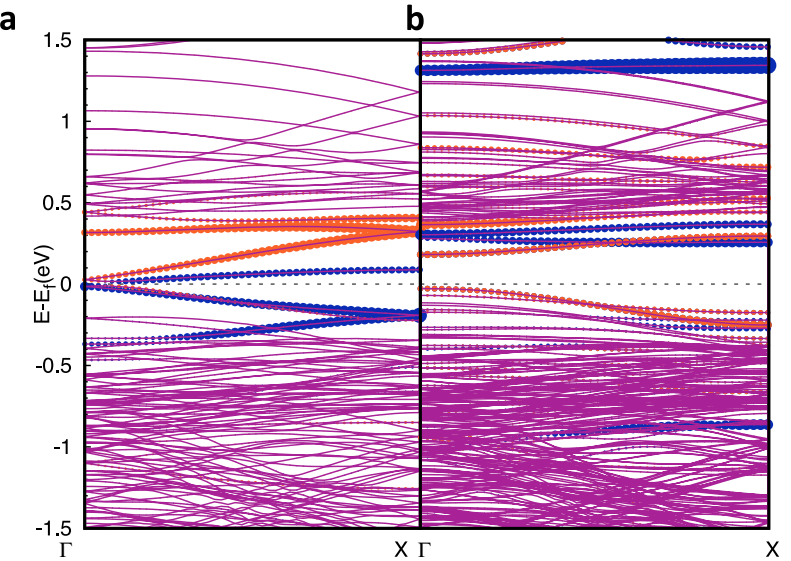

C

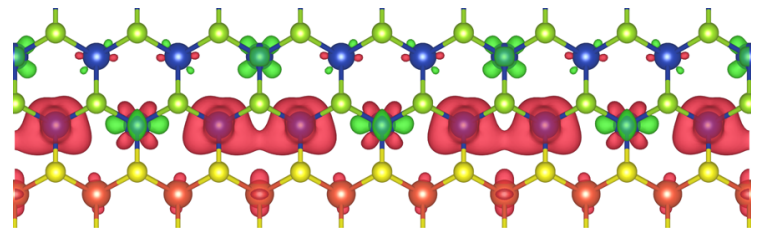

d

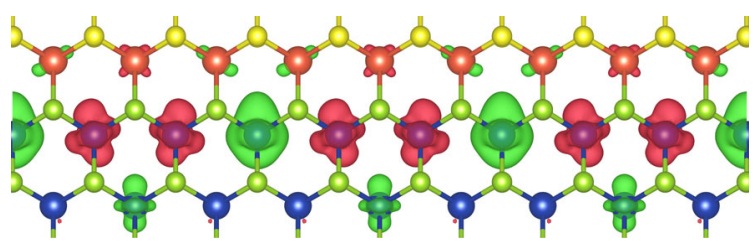

Fig. 3 The band structure of the $\mathrm{H}-\mathrm{MoSe}_{2}$ and $\mathrm{H}-\mathrm{TiS}_{2}$ heterojunction calculated in the $3 \times$ supercells. The states highlighted in blue and red belong to Mo and Ti edges, respectively. a Calculated with the PBE functional, $\mathbf{b}$ with the $\mathrm{PBE}+\mathrm{U}$ functional, both after structural relaxation. The latter gives an FM/AFM SDWs at the Mo/Ti edges, creating gaps of $0.45 / 0.22 \mathrm{eV}$, respectively. c Spin-density wave at the Mo edge and $\mathbf{d}$ at the $\mathrm{Ti}$ edge; the red/green colors indicate spin up/down, where the SDWs result in the three Mo atoms at the junction becoming inequivalent ${ }^{45}$. structure of the $3 \times$ structure. Although the electronic structure close to the Fermi level looks complicated, with multiple bands crossing, these bands are actually the same as the ones shown in Fig. $1 \mathrm{~b}$, but folded because of the $3 \times$ periodicity. The Mo edge band, which is $2 / 3$ occupied in the $1 \times$ cell, is then folded into three bands, with the two lower bands fully occupied, and the topmost one fully empty. The Ti edge band, $1 / 3$ occupied in the $1 \times$ cell, is folded similarly, with the lowest band fully occupied, and the two upper ones fully empty.

The metallic edge bands in Fig. 3a display the prototypical 1D band structure that is susceptible to perturbations inducing a metal-insulator transition, such as a Peierls distortion, or a CDW/ SDW, but a DFT calculation using the PBE functional does not find any of these. The 1D states have mostly Mo $d$ character, and although the on-site electron-electron Coulomb interaction in $4 d$ transition metals is weaker than in $3 d$ ones, it is not always negligible ${ }^{36,37}$. In previous calculations on mirror twin boundaries of $\mathrm{MoSe}_{2}$, we have found that the inclusion of on-site Coulomb and exchange interactions as in the PBE $+U$ mean-field approach markedly changes the electronic structure of the 1D states.

Therefore, we repeat the calculations in the $3 \times$ cells, using the $\mathrm{PBE}+\mathrm{U}$ functional with $U-J=3 \mathrm{eV}$ for the Mo $4 d$ electrons $^{36,37}$. In principle, one can also include such a parameter for the $\mathrm{Ti} 3 d$ electrons, but this has little effect, as in $\mathrm{TiS}_{2}$ these states are mostly empty. The top of the $\mathrm{TiS}_{2}$ valence band has sulfur $p$ character, and the Ti $3 d$ states only contribute significantly to the conduction band. We have also tested values of $U-J$ over the whole range $0-3 \mathrm{eV}$; details can be found in Supplementary Fig. 2.

The $\mathrm{PBE}+\mathrm{U}$ functional indeed gives rise to the opening of a bandgap in the band structure, as shown in Fig. 3b. It is caused by SDWs at both the Mo and Ti edge junctions. In these SDWs, the Mo atoms closest to the junctions carry a magnetic moment, whereas the Ti atoms at the junctions, and the atoms further away from the junctions, do not show any magnetic moments. Several (meta)stable magnetic configurations of Mo atoms at the two interfaces are found. The configuration with the lowest total energy has a ferromagnetic arrangement at the Mo edge, with magnetic moments of 1.0,1.0, and $0.0 \mu_{\mathrm{B}}$ on the three Mo atoms at the junction. An antiferromagnetic arrangement is $60 \mathrm{meV} / 3 \times$ cell higher in energy. At the Ti edge, we found the AFM arrangement to be the lowest in energy, with magnetic moments on the three Mo atoms at the junction of $-1.0,+0.6$, and $+0.6 \mu_{\mathrm{B}}$.

The SDWs are accompanied by CDWs and (slight) distortions of the structure at the junctions. The Mo atoms at the Mo edge adopt a $3 \times$ periodicity, with distances between the Mo atoms of 3.27 and $3.41 \AA$, whereas the distances between the Ti atoms at the Mo edge do not change much. At the Ti edge, the distances between Ti atoms are 3.29 and $3.33 \AA$, and there are no significant changes in the bond distances between the Mo atoms.

The SDW/CDWs lead to a metal-insulator transition, resulting in a bandgap in both the spin configurations (Fig. 3b). The bandgap at the Mo edge is $0.45 \mathrm{eV}$, whereas at the Ti edge, it is $0.22 \mathrm{eV}$. The SDW/CDWs lower the total energy by $330 \mathrm{meV} / 3 \times$ cell compared to the undistorted structure. The exact value of $U-J$ is not too critical for the emergence of an SDW/CDW and a gap. The SDW/ CDW persists for values of $U-J$ in the range $2-3 \mathrm{eV}$, although the magnetic moments and the bandgap decrease on decreasing $U-$ $J$, see Supplementary Fig. 2. The correlation-driven SDW/CDWs seem to be a unique feature of 2D TMDC junctions, due to the presence of $d$ electrons on the transition metals. In the AIN-SiC case, where $d$ electrons are absent, spin-polarized DFT calculations do not give SDW/CDWs, and the junctions remain metallic ${ }^{24}$.

In the case of the $\mathrm{H}-\mathrm{MoTe}_{2} / \mathrm{T}-\mathrm{HfS}_{2}$ junction, simply optimizing the $3 \times$ structure with the PBE functional gives a distinct CDW with a clear $3 \times$ modulation of the structure at both the junctions. The modulation is largest at the Mo edge, with distances between the Mo atoms at the edge becoming 3.39 and $4.11 \AA$. At the Hf edge, the modulation is somewhat smaller, with distances between the 
a

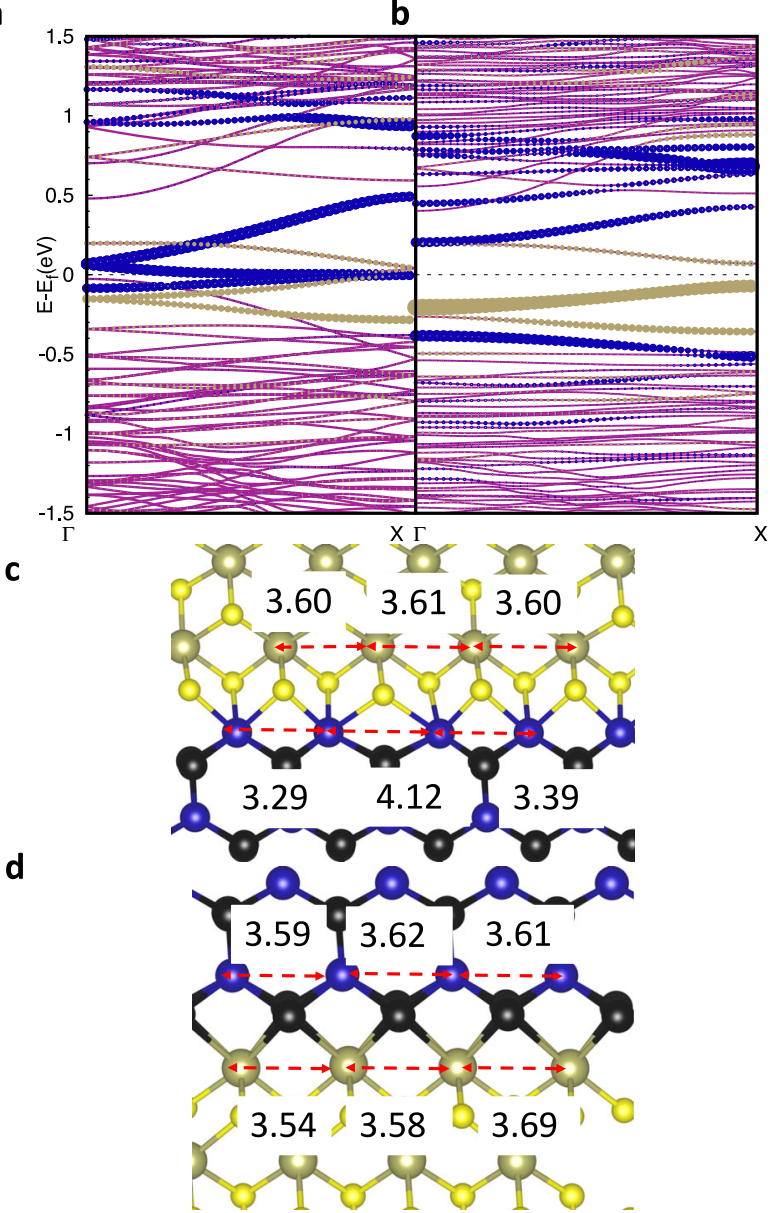

Fig. 4 The band structure of the $\mathrm{H}-\mathrm{MoTe}_{2}$ and $\mathrm{T}-\mathrm{HfS} \mathrm{S}_{2}$ heterojunction calculated in the $3 \times$ cell. The states highlighted in blue and brown belong to Mo and $\mathrm{Hf}$ edges, respectively. a Undistorted $1 \times$ structure. b Peierls distorted structures; bandgaps at the $\mathrm{Hf}$ and Mo edges are 0.15 and $0.6 \mathrm{eV}$, respectively. c, d Peierls distorted structures at Mo and $\mathrm{Hf}$ edges, respectively; the numbers give the distances in $\AA$ between metal atoms at the interfaces.

$\mathrm{Hf}$ atoms at the edge becoming 3.54 and $3.69 \AA$. These Peierls distortions open up a gap of $0.6 \mathrm{eV}$ at the Mo edge and $0.15 \mathrm{eV}$ at the $\mathrm{Hf}$ edge, and lower the energy by $270 \mathrm{meV} / 3 \times$ cell. The band structures of the undistorted and the distorted structures are shown in Fig. 4. Adding spin polarization has no effect, but upon adding on-site interactions, with $U-J=1.5 \mathrm{eV}$, magnetic moments of $-0.48,0.54$, and $-0.30 \mu_{\mathrm{B}}$ develop on the Mo atoms at the Mo edge, whereas the $\mathrm{Hf}$ edge remains unpolarized. The bandgaps change only slightly upon adding $\mathrm{U}$; at the $\mathrm{Hf}$ edge, it is $0.17 \mathrm{eV}$, and at the Mo edge, it is $0.42 \mathrm{eV}$, see Supplementary Fig. 6 for the corresponding band structures.

\section{DISCUSSION}

We have shown by means of first-principles DFT calculations that lateral heterojunctions of $2 \mathrm{D}$ semiconducting TMDCs can have $1 \mathrm{D}$ metallicity in a $1 \times$ unit cell. The metallicity arises out of a discontinuous topological invariant at the junction, the in-plane electric polarization, which is nonzero for TMDCs in the $\mathrm{H}$ structure. Such heterojunctions can be made from two semiconducting TMDCs, both in the $\mathrm{H}$ phase, with different values for the topological invariant, and matching lattice constants. Alternatively, junctions can be made between a TMDC in the $\mathrm{H}$ structure with nonzero polarization and one in the T structure with zero polarization, again with matching lattice constants. Changing the chalcogen atoms or creating defects at the junctions will not affect the invariant and hence metallicity is preserved.

The 1D metallic states are, however, susceptible to the instabilities of $1 \mathrm{D}$ metals originating from electron-electron and electron-lattice interactions. Using DFT $+U$ calculations, we show that spin- and/or charge-density waves create a bandgap at the junction, which is, however, much smaller than the bandgaps of the two TMDC semiconductors. The details of these density waves depend upon the detailed structure of the junction. We propose that 2D TMDC heterojunctions are ideal systems for studying 1D physics.

\section{METHODS \\ Density functional theory}

To model a 2D TDMC heterojunction, we build a supercell that has a width of twelve $\mathrm{MX}_{2}$ unit cells, six for each of the two compounds forming the junction. This unit is periodically repeated in plane, such that each supercell contains two junctions, see, for instance, Fig. 1a. Perpendicular to this $2 \mathrm{D}$ plane, we use a vacuum spacing of $15 \AA$ to prevent an interaction between the periodic images.

We perform DFT calculations, with the generalized gradient approximation PBE and PBE $+U$ functionals, and the projector augmented wave method, using the Vienna ab initio simulation package (VASP) ${ }^{38-44}$. For the transition metals in $\mathrm{MX}_{2}$, the outer $s, p$, and $d$ shells are treated as valence electrons, and for the chalcogen atoms, the outer $s$ and $p$ shells. A cut-off of $400 \mathrm{eV}$ for the kinetic energy of the plane waves and a $k$-point sampling of 12 points per unit cell along the direction of the junction are used. All atomic positions along with the lattice constants are relaxed, till the forces on the atoms are $<0.05 \mathrm{eV} / \AA$, with a total energy convergence criterion of $10^{-5} \mathrm{eV}$.

For the $\mathrm{PBE}+U$ calculations, we use the rotationally averaged formulation, as implemented in VASP, which applies a single parameter $U-J^{44}$. We use a value $U-J=3 \mathrm{eV}$, which is appropriate for $4 d$ transition metals, and test values between 0 and $3 \mathrm{eV}^{36,37}$.

\section{DATA AVAILABILITY}

The authors declare that all data supporting the findings of this study are available within the paper and its Supplementary information files.

Received: 15 November 2020; Accepted: 16 March 2021; Published online: 16 April 2021

\section{REFERENCES}

1. Gong, Y. et al. Vertical and in-plane heterostructures from $\mathrm{WS}_{2} / \mathrm{MoS}_{2}$ monolayers. Nat. Mater. 13, 1135-1142 (2014).

2. Ávalos-Ovando, O., Mastrogiuseppe, D. \& Ulloa, S. E. Lateral heterostructures and one-dimensional interfaces in $2 \mathrm{~d}$ transition metal dichalcogenides. J. Condens. Matter Phys. 31, 213001 (2019).

3. Zhang, C. et al. Strain distributions and their influence on electronic structures of $\mathrm{WSe}_{2}-\mathrm{MoS}_{2}$ laterally strained heterojunctions. Nat. Nanotechnol. 13, 152-158 (2018).

4. Huang, C. et al. Lateral heterojunctions within monolayer $\mathrm{MoSe}_{2}-\mathrm{WSe}_{2}$ semiconductors. Nat. Mater. 13, 1096-1101 (2014).

5. Levendorf, M. P. et al. Graphene and boron nitride lateral heterostructures for atomically thin circuitry. Nature 488, 627-632 (2012).

6. Li, M.-Y. et al. Epitaxial growth of a monolayer $\mathrm{WSe}_{2}-\mathrm{MoS}_{2}$ lateral p-n junction with an atomically sharp interface. Science 349, 524-528 (2015).

7. Lin, Y.-C., Dumcenco, D. O., Huang, Y.-S. \& Suenaga, K. Atomic mechanism of the semiconducting-to-metallic phase transition in single-layered $\mathrm{MoS}_{2}$. Nat. Nanotechnol. 9, 391-396 (2014).

8. Ling, X. et al. Parallel stitching of 2D materials. Adv. Mater. 28, 2322-2329 (2016).

9. Nguyen, V.-H. et al. Recent advances in two-dimensional transition metal dichalcogenides as photoelectrocatalyst for hydrogen evolution reaction. J. Chem. Technol. Biotechnol. 95, 2597-2607 (2020).

10. Jaramillo, T. F. et al. Identification of active edge sites for electrochemical $\mathrm{H}_{2}$ evolution from $\mathrm{MoS}_{2}$ nanocatalysts. Science 317, 100-102 (2007).

11. Ponraj, J. S. et al. Photonics and optoelectronics of two-dimensional materials beyond graphene. Nanotechnology 27, 462001 (2016). 
12. Gibertini, M., Pizzi, G. \& Marzari, N. Engineering polar discontinuities in honeycomb lattices. Nat. Commun. 5, 5157 (2014).

13. Gibertini, M. \& Marzari, N. Emergence of one-dimensional wires of free carriers in transition-metal-dichalcogenide nanostructures. Nano Lett. 15, 6229-6238 (2015).

14. Fang, C., Gilbert, M. J. \& Bernevig, B. A. Bulk topological invariants in noninteracting point group symmetric insulators. Phys. Rev. B 86, 115112 (2012).

15. Jadaun, P., Xiao, D., Niu, Q. \& Banerjee, S. K. Topological classification of crystalline insulators with space group symmetry. Phys. Rev. B 88, 085110 (2013).

16. Barja, S. et al. Charge density wave order in $1 \mathrm{~d}$ mirror twin boundaries of singlelayer $\mathrm{MoSe}_{2}$. Nat. Phys. 12, $751 \mathrm{EP}-$ (2016).

17. Ma, Y. et al. Angle resolved photoemission spectroscopy reveals spin charge separation in metallic $\mathrm{MoSe}_{2}$ grain boundary. Nat. Commun. 8, $14231 \mathrm{EP}$ - (2017).

18. Jolie, W. et al. Tomonaga-luttinger liquid in a box: Electrons confined within $\mathrm{MoS}_{2}$ mirror-twin boundaries. Phys. Rev. X 9, 011055 (2019).

19. Bollinger, M. V. et al. One-dimensional metallic edge states in $\mathrm{MoS}_{2}$. Phys. Rev. Lett. 87, 196803 (2001).

20. Komsa, H.-P. \& Krasheninnikov, A. V. Engineering the electronic properties of twodimensional transition metal dichalcogenides by introducing mirror twin boundaries. Adv. Electron. Mater. 3, 1600468 (2017).

21. Güller, F., Llois, A. M., Goniakowski, J. \& Noguera, C. Polarity effects in unsupported polar nanoribbons. Phys. Rev. B 87, 205423 (2013).

22. Krishnamurthi, S. \& Brocks, G. Spin/charge density waves at the boundaries of transition metal dichalcogenides. Phys. Rev. B 102, 161106 (2020).

23. Krishnamurthi, S., Farmanbar, M. \& Brocks, G. One-dimensional electronic instabilities at the edges of MoS 2 . Phys. Rev. B 102, 165142 (2020).

24. Bristowe, N. C., Stengel, M., Littlewood, P. B., Artacho, E. \& Pruneda, J. M. Onedimensional half-metallic interfaces of two-dimensional honeycomb insulators. Phys. Rev. B 88, 161411 (2013).

25. Chen, G.-X., Li, X.-G., Wang, Y.-P., Fry, J. N. \& Cheng, H.-P. Two-dimensional lateral $\mathrm{GaN} / \mathrm{SiC}$ heterostructures: First-principles studies of electronic and magnetic properties. Phys. Rev. B 95, 045302 (2017).

26. Choi, J.-H., Liu, S., Zhang, W., Liu, Z. \& Rummeli, M. H. Charge density waves driven by peierls instability at the interface of two-dimensional lateral heterostructures. Small 14, 1803040 (2018).

27. King-Smith, R. D. \& Vanderbilt, D. Theory of polarization of crystalline solids. Phys. Rev. B 47, 1651-1654 (1993).

28. Wang, H., Wei, W., Li, F., Huang, B. \& Dai, Y. Electronic and magnetic properties of the one-dimensional interfaces of two-dimensional lateral GeC/BP heterostructures. Phys. Chem. Chem. Phys. 21, 8856-8864 (2019).

29. Mounet, N. et al. Two-dimensional materials from high-throughput computational exfoliation of experimentally known compounds. Nat. Nanotechnol. 13, 246-252 (2018).

30. Eda, G. et al. Coherent atomic and electronic heterostructures of single-layer $\mathrm{MoS}_{2}$. ACS Nano 6, 7311-7317 (2012).

31. Ohtomo, A. \& Hwang, H. Y. A high-mobility electron gas at the $\mathrm{LaAlO}_{3} / \mathrm{SrTiO}_{3}$ heterointerface. Nature 427, 423-426 (2004).

32. Nakagawa, N., Hwang, H. Y. \& Muller, D. A. Why some interfaces cannot be sharp. Nat. Mater. 5, 204-209 (2006).

33. Farmanbar, M., Amlaki, T. \& Brocks, G. Green's function approach to edge states in transition metal dichalcogenides. Phys. Rev. B 93, 205444 (2016).

34. Mortazavi, B. \& Rabczuk, T. Multiscale modelling of heat conduction in all-MoS 2 single-layer heterostructures. RSC Adv. 7, 11135-11141 (2017).

35. Lucking, M. C., Bang, J., Terrones, H., Sun, Y.-Y. \& Zhang, S. Multivalency-induced band gap opening at $\mathrm{MoS}_{2}$ edges. Chem. Mater. 27, 3326-3331 (2015).

36. Meredig, B., Thompson, A., Hansen, H. A., Wolverton, C. \& van de Walle, A. Method for locating low-energy solutions within DFT + U. Phys. Rev. B 82, 195128 (2010).

37. Vaugier, L., Jiang, H. \& Biermann, S. Hubbard $U$ and hund exchange $J$ in transition metal oxides: Screening versus localization trends from constrained random phase approximation. Phys. Rev. B 86, 165105 (2012).

38. Kresse, G. \& Furthmüller, J. Efficient iterative schemes for ab initio total-energy calculations using a plane-wave basis set. Phys. Rev. B 54, 11169-11186 (1996).

39. Kresse, G. \& Joubert, D. From ultrasoft pseudopotentials to the projector augmented-wave method. Phys. Rev. B 59, 1758-1775 (1999).
40. Blöchl, P. E. Projector augmented-wave method. Phys. Rev. B 50, 17953-17979 (1994).

41. Hohenberg, P. \& Kohn, W. Inhomogeneous electron gas. Phys. Rev. 136, B864-B871 (1964).

42. Kohn, W. \& Sham, L. J. Self-consistent equations including exchange and correlation effects. Phys. Rev. 140, A1133-A1138 (1965).

43. Perdew, J. P. \& Zunger, A. Self-interaction correction to density-functional approximations for many-electron systems. Phys. Rev. B 23, 5048-5079 (1981).

44. Dudarev, S. L., Botton, G. A., Savrasov, S. Y., Humphreys, C. J. \& Sutton, A. P. Electron-energy-loss spectra and the structural stability of nickel oxide: an LSDA +U study. Phys. Rev. B 57, 1505-1509 (1998).

45. Momma, K. \& Izumi, F. VESTA3 for three-dimensional visualization of crystal, volumetric and morphology data. J. Appl. Crystallogr 44, 1272-1276 (2011).

\section{ACKNOWLEDGEMENTS}

This work was financially supported by the "Nederlandse Organisatie voor Wetenschappelijk Onderzoek" (NWO) through the research program of the former "Stichting voor Fundamenteel Onderzoek der Materie" (NWO-I, formerly FOM) and through the use of supercomputer facilities of NWO "Exacte Wetenschappen" (Physical Sciences). We acknowledge the funding from the Shell-NWO/FOM Computational Sciences for Energy Research program (Project No. 15CSER025)

\section{AUTHOR CONTRIBUTIONS}

G.B. conceived the idea and S.K. performed the DFT calculations. Both authors were involved in discussing the results and in writing the manuscript.

\section{COMPETING INTERESTS}

The authors declare no competing interests.

\section{ADDITIONAL INFORMATION}

Supplementary information The online version contains supplementary material available at https://doi.org/10.1038/s41699-021-00224-1.

Correspondence and requests for materials should be addressed to G.B.

Reprints and permission information is available at http://www.nature.com/ reprints

Publisher's note Springer Nature remains neutral with regard to jurisdictional claims in published maps and institutional affiliations.

\begin{abstract}
(c) Open Access This article is licensed under a Creative Commons Attribution 4.0 International License, which permits use, sharing, adaptation, distribution and reproduction in any medium or format, as long as you give appropriate credit to the original author(s) and the source, provide a link to the Creative Commons license, and indicate if changes were made. The images or other third party material in this article are included in the article's Creative Commons license, unless indicated otherwise in a credit line to the material. If material is not included in the article's Creative Commons license and your intended use is not permitted by statutory regulation or exceeds the permitted use, you will need to obtain permission directly from the copyright holder. To view a copy of this license, visit http://creativecommons. org/licenses/by/4.0/.
\end{abstract}

(c) The Author(s) 2021 